\title{
Same-Session Bilateral Ureteroscopy: Safety and Outcomes
}

\section{Urology}

Johann P. Ingimarsson MD ${ }^{1}$, Marcelino Rivera $\mathrm{MD}^{2}$, John J. Knoedler MD ${ }^{3}$, Amy E. Krambeck $\mathrm{MD}^{2}$

1: Maine Medical Center, Department of Urology, Portland, Maine and Tufts School of Medicine, Boston Massachusetts, USA, 2 University of Indiana, Department of Urology, Indianapolis, Indiana, USA, 3 Mayo Clinic, Department of Urology, Rochester, Minnnesota, USA.

Correspondence:

Johann P. Ingimarsson MD, Clinical Assistant Professor

Maine Medical Center Urology, 100 Brickhill Avenue, South Portland, Maine, 04106, United States of America

E-mail: JIngimarss@mmc.org, Tel: 207- 773-1728, Fax: 207-773-8153
Abstract: 250 words
Main text: 2679 words
Tables: 2 Figures: None
References:

27

Keywords: Ureteroscopy, bilateral, stones, complications, outcomes

This is the author's manuscript of the article published in final edited form as:

Ingimarsson, J. P., Rivera, M., Knoedler, J. J., \& Krambeck, A. E. (2017). Same-Session Bilateral Ureteroscopy: Safety and Outcomes. Urology. https://doi.org/10.1016/j.urology.2017.06.027 


\begin{abstract}
:
Objective: To assess the complications and outcomes associated with same-session bilateral ureteroscopy in a tertiary referral center, as same-session bilateral ureteroscopy for stone disease has been critiqued for the theoretical risk of injury to both ureters with subsequent risk to renal function.
\end{abstract}

Methods: We retrospectively reviewed all cases of bilateral ureteroscopy performed for urolithiasis by a single surgeon at out institution between 2009 and 2014 . These were compared to a prospective unilateral ureteroscopy data base

Results: There were 117 same-session bilateral ureteroscopic procedures performed in 113 patients totaling 234 ureteroscopies. A flexible ureteroscope was uses in 228 ureters (97.4\%), and $6(2.6 \%)$ were semi-rigid only. Ureteral dilators were required in $8(6.8 \%)$ cases. Pre stenting was performed in 23 patients $(19.6 \%)$.

Short-term complications were observed following 19 procedures $(16.2 \%)$ including: $11(9.4 \%)$ Clavien I, 4 (3.4\%) Clavien II and 4 (3.4\%) Clavien III. Of the 84 (71.8\%) patients that completed 6 week follow up there were no long term complications. Stone free rates were 91.4\% for patients imaged with KUB and US, and $84.2 \%$ for those with CT scans. . Neither complications nor re-admissions were significantly different in the unilateral group. Median length of follow up for the entire cohort was 2.8 years (range 0 - 7 years).

Conclusions: Bilateral ureteroscopy can be performed safely with short-term complications consistent with published literature. We found no long-term complications and high stone free rates. Bilateral ureteroscopy in a single procedure represents a viable standard of care for patients with bilateral stone disease.

\title{
Objective:
}


Stone patients commonly present with multiple and bilateral stones $(1,2)$. In order to limit missed time from work and multiple anesthetics, some patients desire treatment of all stones, including those in both kidneys, in one setting. Shock wave lithotripsy, while a good option for small to moderate sized solitary urolithiasis, has been shown to be associated with worse stonefree and retreatment rates, especially with multiple stones, when compared to ureteroscopy (3, 4). Ureteroscopy has good reported stone free rates, but does carry a known risk of infection and ureteral injury of $10-16 \%$ (5). Therefore, performing a same session bilateral ureteroscopy, while more convenient for the patient, theoretically doubles the risk to the patient and subjects both kidneys to potentially serious consequences. In fact, an earlier study found a $29 \%$ risk of complications of bilateral ureteroscopy, substantially higher than in staged bilateral procedures (6). More recent studies have reported lower rates of and less severe complications, but many of these series are small cohorts with various different surgical techniques (6-14). In order to address the concerns for short and long-term complications associated with bilateral ureteroscopy we evaluated a large cohort, performed by a single surgeon series in the modern era. The aim of this study was to address clinical outcomes of same-session bilateral ureteroscopy.

\section{Methods:}

After Institutional Review Board approval, a retrospective chart review was performed. Included were all cases of bilateral ureteroscopy performed for urolithiasis by a single surgeon at out institution between January 2009 and December 2014 for stone disease. This data included patients with prior stenting, either in the emergent setting due to intractable pain or infection, or if prior ureteroscopy had failed due to tight ureter. Bilateral ureteroscopy for indications other 
than stone disease were excluded. Scheduled staged bilateral ureteroscopies were not done at our institutions. Variables recorded included demographics, indications for surgery, prior stone history, imaging findings including stones size and location; complications, stone free states, preand post- operative creatinine and stone chemical composition. Furthermore, hospital admissions, emergency department visits, re-admissions, repeat surgeries and patient initiated phone calls in the first 30 days after surgery were recorded.

Complications were compared to an IRB approved prospectively collected data base of unilateral ureteroscopies performed over the same time interval.

All patients had pre-operative computed tomography (CT) scans and urine cultures. Stone size was recorded as largest stone diameter in any plane. Patients received antibiotics tailored to a pre-operative urine culture. If the urine culture was negative, one dose of oral ciprofloxacin was administered one hour prior to anesthesia unless the patient was allergic to fluoroquinolones, then an alternate agent was administered. Retrograde pyelogram was performed in all cases. Routine ureteral dilation was not performed; however, if a localized area of narrowing was noted on retrograde pyelogram then sequential plastic disposable ureteral dilators were utilized to facilitate scope or access sheath passage, usually to 14 Fr. Distal stones were treated with a 6 Fr semi rigid ureteroscopes. Renal stones, as well as, those in the proximal or mid ureter were treated with a 7 or 8 Fr flexible ureteroscope with an access sheaths, most often 12/14 or 11/13 Fr. Pressurized irrigation was routinely used in the setting of an access sheath. Stone fragmentation was performed using the holmium laser with a 200 micrometer laser fiber and all fragments were removed using a nitinol basket. Bilateral ureteral stents were typically left for 72 hours on a dangle extraction string for patients to remove themselves after ureteroscopy. If there 
were any concerns for ureteral perforation, residual fragments or significant edema, the extraction string was removed and the stent was left indwelling for two weeks.

Routine patient followed up was at 6 weeks postoperatively with a renal ultrasound (US) and an X-ray of kidney ureter and bladder (KUB). In certain patients, a post- operative CT scan was obtained, if the patient was suspected of having uric acid stones intraoperatively, needed imaging for another reason such as an unrelated cancer surveillance or if the patient described persistent postoperative pain. Otherwise a KUB and ultrasound were obtained. Stone free was defined as absence of any stone fragments on KUB and US, or on CT scans. Short-term complications were defined as occurring <30 days from surgery. Complications were categorized by the ClavienDindo classification (15). Ureteral injuries were graded by the grading system proposed by Traxer and Thomas (16). Later encounters within our health system were reviewed to assess for long term complications, defined as new onset or worsening chronic kidney injury or ureteral stricture disease as documented by new hydronephrosis on ultrasound or CT scan. Longer term follow up was not standardized but constituted of any combination of serum chemistries, ultrasound, KUB and computed tomography.

Student t-test was used to compare means and Fisher's exact, two tailed to compare number of events and a p-value of $<0.05$ considered significant.

\section{Results:}

There were 117 same-session bilateral ureteroscopic procedures performed among 113 patients totaling 234 renal units. Fifty seven ureters were treated for stones and 213 kidneys (see table 1). Median age of the patients undergoing bilateral ureteroscopy was 56 years (SD 16 IQR 43-65), median BMI was 29 (STD 7, IQR 26-35) and 58 (49.6\%) were female. Prior stone history was 
evident in $92(78.6 \%)$ of patients. Mean preoperative creatinine was 0.99 (SD 0.28). Chronic kidney disease stage 3 was present in 19 patients and none had stage 4 or 5 .

For the bilateral group, we performed 25 procedures $(21.4 \%)$ for incident stones and $92(78.6 \%)$ for recurrent stones. Renal colic was present in 65 (56\%) patients, on the left side in 47 (40.2\%) and on the right in $39(33.3 \%)$. Bilateral ureteral stones were present in 4 (3.4\%). Median renal stone size was $6.9 \mathrm{~mm}$ (SD $4.4 \mathrm{~mm}$, IQR 4-9 mm) and median ureteral stone size was $7.0 \mathrm{~mm}$ (SD $3.1 \mathrm{~mm}$, IQR 4-8mm). Stone volume was a median of $905 \mathrm{~mm}^{3}$. The predominant stone composition was calcium oxalate in $83(71 \%)$ cases, apatite in $21(18 \%)$, brushite in $6(5 \%)$, uric acid in $5(4 \%)$ and ammonium urate in $2(2 \%)$. Pre-stenting was necessary in 15 patients $(12.8 \%)$ in the bilateral cohort and ureteral dilation was required in $8(6.8 \%)$ cases. A flexible ureteroscope with access sheath was used in 228 (97.4\%) ureters and a semirigid ureteroscope only in $6(2.4 \%)$ ureters.

Intraoperatively, ureteral injury was identified in 5 of $234(2.1 \%)$ ureters in the bilateral ureteroscopy cohort. Of these, 3 were superficial injuries (grade I) noted at completion of the procedure and treated with a stenting for 2 weeks. Both the grade II and grade III were identified early in the case resulting in abortion of the procedure with stent placement and return in 2 weeks for completion of the ureteroscopic procedure.

The majority of patients were discharged to home the day of surgery. Patients were admitted overnight following the surgery in 23 procedures (19.7\%), of which $10(8.5 \%)$ were previously scheduled admissions for observation due to advanced age or significant medical comorbidities. 
Of the remaining $13(11.2 \%)$ unscheduled admissions, 10 patients were admitted for pain control, 2 were for nausea, and 1 for social concerns.

Unscheduled emergency department visits occurred in 14 (12.0\%) patients within 30 days of surgery. These visits included: 8 for flank pain after stent removal of which 2 required reinsertion of the stents for 2 weeks, 3 for fevers with urinary tract infection (all admitted), 1 with hyponatremia and ileus (admitted), 1 with urinary retention and 1 with urge incontinence (neither admitted). Additionally, 23 (19.7\%) other patients called our department postoperatively, $10(8.5 \%)$ with complaints of stent pain or discomfort and $13(11.1 \%)$ for pain following stent removal and were managed conservatively.

In total, short-term complications were observed following 19/117 (16.2\%) bilateral ureteroscopic procedures. These are listed in table 2. These included $11(9.4 \%)$ Clavien I, 4 (3.4\%) Clavien II and 4 (3.4\%) Clavien III complications. Patients in the bilateral cohort who suffered complications did not significantly differ from those who did not with regards to age, gender, body mass index, prior stone history, prior ureteroscopy history, prior stenting, symptomatic presentation, stone composition, stone location or intra-operative ureteral dilation (all p-values $>0.3$ ). Therefore multivariate analysis was not performed.

Further, in the bilateral group, while no variable was statistically significant when comparing injured ureters to those not injured, it should be noted that none of the injured ureters had been pre-stented where as $17.1 \%$ of non-injured ureters had been $(\mathrm{p}=0.37)$. Dilation was performed in 1 of $5(20.0 \%)$ of injured ureters, whereas 7 of the 229 non injured ureters $(3.1 \%)$ did require dilation $(\mathrm{p}=0.16)$ and 2 of $5(40.0 \%)$ injured ureters were operated on for ureteral stones, compared to $47(20.5 \%)$ of ureters without injury $(\mathrm{p}=0.28)$. 
Data from routine 6 week follow up was available in $84(71.8 \%)$ patients who underwent bilateral ureteroscopy. Excluding 7 patients with known intraparenchymal stones, nephrocalcinosis or medullary sponge kidneys which had expected calcifications on post op imaging, 77 patients had follow up imaging at week 6 for review. Thereof, 116 renal units were imaged with KUB and US and 38 renal units with CT. Renal unit stone free rate was 104 $(89.8 \%)$ after first procedure, with 2 patients undergoing a second ureteroscopy with a resulting 91.4\% final stone free rate in the KUB/US group. In the CT group 30 (78.8\%) renal units were stone free after the initial procedure with 2 patients undergoing a second ureteroscopy with a resulting $84.2 \%$ final stone free rate.

At 6 week follow-up there were no complications such as stricture, new-onset hydronephrosis, renal scarring, or change in creatinine $>0.2 \mathrm{mg} / \mathrm{dL}$ from baseline. Pre- and post- operative creatinine was available in 94 patients $(80.3 \%)$. Mean creatinine was 0.99 (SD 0.28) preoperative and 1.00 (SD 0.29) post op. One patient, with known Bartter's syndrome, suffered acute kidney injury perioperatively that resolved (table 2). Additional follow-up of a mean of 2.8 years (range 0-7 years) revealed no further long term renal insufficiency caused by a surgical complication. However, end stage renal failure developed in 2 patients at 4 and 5 years postoperatively, respectively. The cause for renal failure was attributed to their underlying disease process, one with primary hyperoxaluria type I and the other, the above mentioned patient with Bartter's syndrome; neither demonstrated any signs of hydronephrosis or ureteral stricture.

Certain variables were compared to a database of 134 unilateral ureteroscopies performed over the same time period. The p-values in the following text denote comparison with the same variable in bilateral group. Median age in the unilateral group was 58 (SD 14, IQR 49-68) 
$(\mathrm{p}=0.88)$ and $51.9 \%$ were female. Among the unilateral ureteroscopy the median renal stone size was $7.5 \mathrm{~mm}$ (SD $4.7 \mathrm{~mm}$, IQR 4-10 mm), which was not significantly different than the bilateral cohort $(\mathrm{P}=0.42)$. Median ureteral stone size was $7.9 \mathrm{~mm}$ (SD $3.9 \mathrm{~mm}$, IQR 5-10mm), which was also similar to the bilateral cohort $(\mathrm{p}=0.29)$. Stone volume was a median of $539 \mathrm{~mm} 3$ in the unilateral group, and was also not significantly different than the bilateral cohort $(\mathrm{p}=0.11)$. When comparing complications, there was only 1 intraoperative perforation in the unilateral ureteroscopy group $(0.75 \%)$, which was grade III and this difference was not statistically lower than the bilateral cohort $(\mathrm{p}=0.42)$. Unscheduled emergency room visits and or 30 day readmissions in the unilateral cohort occurred in 16 of the $133(12.0 \%)$, similar to the bilateral cohort $(\mathrm{p}=0.98)$. Postoperative complications occurred in $15.8 \%$ of the unilateral ureteroscopy patients, where $13(9.8 \%)$ had Clavien I,5 (3.8\%) had Clavien II and $3(2.2 \%)$ had Clavien III complications. The postoperative complication rate did not differ from the bilateral ureteroscopy cohort $(\mathrm{p}=0.79)$. At 6 week follow the stone free rate by KUB and ultrasound was $74 \%$, which was lower than the bilateral group; however patients with known nephrocalcinosis were not excluded in this prospective database. No patient in the unilateral cohort developed ureteral stricture or hydronephrosis.

\section{Discussion:}

Our study demonstrates that bilateral ureteroscopy can be performed with a low likelihood $(6.8 \%)$ of serious complications (Clavien II or higher) and no long term adverse events. It further 
demonstrates that this rate is not significantly higher than complications of unilateral ureteroscopies. None of the patients in the bilateral cohort develop ureteral stricture disease or worsening renal function secondary to their bilateral ureteroscopy. With good stone clearance outcomes, it can be concluded that same-session bilateral ureteroscopy is a safe and effective way to treat bilateral urolithiasis. However, there is notable, self limiting short term morbidity. This morbidity is evident by the fact that $9 \%$ of patients had Clavien I complications, primarily emergency department visits for stent pain and discomfort and additionally $20 \%$ of patients called in with complaints of stent discomfort or pain after removing their stents. Thus, bilateral ureteroscopy may result in more short term discomfort and pain compared to a unilateral ureteroscopy. The unscheduled admission rates in our study, 12\%, are clearly higher than previous reported numbers from unilateral ureteroscopy of $4 \%$. (17). However, the emergency room visits rate $(12.0 \%)$ and readmission $(5.1 \%)$ rate in this study did not differ from the unilateral ureteroscopy cohort and are similar to the published rates for unilateral ureteroscopy of $15.6 \%$ and $5.8 \%$ respectively. (18).

The overall complication rate of bilateral ureterosocpy in the current study was $16.2 \%$, which is consistent with prior studies. Published complication rates from prior cohort series of bilateral ureteroscopic procedures ranged from 10-29\%, when accounting for traditionally accepted Clavien I-V complications (6-14). Of note, authors that have recorded less severe morbidity have expectedly noted much higher complication rates. For example, Isen reported $29 \%$ flank pain and $66 \%$ stent related lower urinary tract symptoms (9). Their findings support our results, which found $35(29.9 \%)$ patients either called or presented to an emergency department with such complaints. This contrasts to the recent reported morbidity of unilateral uretersocopy 4-13\% (1821). Because of this, patients might be advised of the increased short-term morbidity associated 
with bilateral ureteroscopy and stenting, as compared to each episode of a staged ureteroscopy, again however, the patients are spared a second ureteroscopy which as our unilateral data would suggest carries similar risk as the first procedure.

The stone free rates in the current study are comparable to published series on bilateral ureteroscopies, which have ranged from $85-99 \%$, depending on imaging modality and definition of stone free state (6-14)., again arguing for the efficacy of same-session bilateral ureteroscopy. The differences noted between the stone free rate in the unilateral cohort and that of the bilateral cohort most likely lies in data collection. We excluded patients with nephrocalcinosis in the bilateral retrospective chart review when assessing stone free results; however, in the prospectively collected unilateral ureteroscopy database these patients are not excluded and any residual calcification noted on the postoperative imaging is recorded. Furthermore this finding compares well to stone free rates of unilateral ureteroscopy, where 70-97\% of patients surveilled with plain radiography and US were stone free (22-24)., and 55-60\% of those surveilled with CT scan (25-27).

The study is limited by its retrospective nature and variation in length of follow up, and a lack of followup beyond the immediate postoperative period in a quarter of patients Furthermore, the comparison to the unilateral database is limited due to data collection differences. None the less, it is reassuring that we identified no long term sequelae such as ureteral stricture or renal function deterioration. Specifically, none of the 5 patients, of which 4 have been followed for greater than 4 years, who had an identified ureteral injury developed ureteral stricture, hydronephrosis or renal insufficiency. Thus, performing bilateral ureteroscopy does not appear to present increased risk of ureteral or renal compromise, which is a common concern with the procedure. 
Conclusions: We present one of the largest series of bilateral ureteroscopy reported to date. Our study demonstrates that bilateral ureteroscopy performed in the same session can be safe and effective with minimal short-term complications, similar to unilateral ureteroscopy Furthermore we found no long-term complications and high overall stone free rates. As such, bilateral ureteroscopy in a single procedure represents a viable standard of care for patients with bilateral stone disease. 
References:

1 Abe T, AK, Kawaguchi M, Ueda T, Ichikawa T, Ito H, Nozumi K, Suzuki K. Outcomes of shockwave lithotripsy for upper urinary tract stones: a large-scale study at a single institution. $\mathrm{J}$ Endourol. 2005;19:768-73.

2 Kanao K, NJ, Nakagawa K, et al. Preoperative nomograms for predicting stone-free rate after extracorporeal shock wave lithotripsy. J Urol. 2006;176:1453-6.

3 Cui X, JF, Yan H, Ou TW, Jia CS, He XZ, Gao W, Wang Q, Cui B, Wu JT. Comparison between extracorporeal shock wave lithotripsy and ureteroscopic lithotripsy for treating large proximal ureteral stones: a meta-analysis. Urology. 2015;85:748-56.

4 Donaldson JF, LM, Scrimgeour D, Stewart F, MacLennan S, Lam TB, McClinton S.

Systematic review and meta-analysis of the clinical effectiveness of shock wave lithotripsy, retrograde intrarenal surgery, and percutaneous nephrolithotomy for lower-pole renal stones. Eur Urol. 2015;67:612-6.

5 Preminger GM1, TH, Assimos DG et al. 2007 guideline for the management of ureteral calculi. J Urol. 2007;178:2418-34.

6 Hollenbeck BK, ST, Faerber GJ, Wolf JS., Jr. Safety and efficacy of same-session bilateral ureteroscopy. J Endourol. 2003;17:881-5.

7 Darabi M, KM. Bilateral same-session ureteroscopy: its efficacy and safety for diagnosis and treatment. Urol J. 2005;2:8-12.

8 El-Hefnawy AS, E-NA, El-Tabey NA, et al. Bilateral same-session ureteroscopy for treatment of ureteral calculi: critical analysis of risk factors. Scand J Urol Nephrol. 2011;45:97-101. 9 K., I. Single-session ureteroscopic pneumatic lithotripsy for the management of bilateral ureteric stones. Int Braz J Urol. 2012;38:63-8.

10 Watson JM, CC, Pattaras JG, Ogan K. Same session bilateral ureteroscopy is safe and efficacious. J Urol. 2011;185:170-4.

11 Gunlusoy B, DT, Arslan M, et al. Is bilateral ureterorenoscopy the first choice for the treatment of bilateral ureteral stones? An updated study. 2012;89:412-7.

12 Mushtaque M, GC, Shah I, Khanday MA, Khanday SA. Outcome of bilateral ureteroscopic retrieval of stones in a single session. Urol Ann.4:158-61.

13 Huang Z, FF, Zhong Z, Zhang L, Xu R, Zhao X. . Flexible ureteroscopy and laser lithotripsy for bilateral multiple intrarenal stones: is this a valuable choice? Urology. 2012;80:800-4. 14 Drake T, AA, Somani BK. Feasibility and safety of bilateral same-session flexible ureteroscopy (FURS) for renal and ureteral stone disease. Cent European J Urol. 2015;68:193-6. 15 Dindo D, DN, Clavien PA. Classification of surgical complications: a new proposal with evaluation in a cohort of 6336 patients and results of a survey. Ann Surg. 2004;240:205-13. 16 Traxer O, TA. Prospective evaluation and classification of ureteral wall injuries resulting from insertion of a ureteral access sheath during retrograde intrarenal surgery. J Urol.

2013;189:580-4.

17 Tan, HS, SA; He, C; Roberts, WW; Faerber, GJ; Wolf, JS Jr:. Immediate unplanned hospital admission after outpatient ureteroscopy for stone disease. J Urol. 2011;185(6):2181-5. .

18 Bloom, JM, G; Phillips, J;. Factors Influencing Readmission after Elective Ureteroscopy. J Urol. 2016;195(5):1487-91.

19 Berardinelli, FC, L; De Francesco, P; Proietti, S; Hennessey, D; Dalpiaz, O; Cracco, CM; Pellegrini, F; Scoffone, CM; Schips, L; Giusti, G. The surgical experience influences the safety 
of retrograde intrarenal surgery for kidney stones: a propensity score analysis. Urolithiasis. 2016; [Epub ahead of print].

20 Singh, VP, B; Sinha, RJ. rospective randomized comparison between fluoroscopy-guided ureteroscopy versus ureteroscopy with real-time ultrasonography for the management of ureteral stones. Urol Ann. 2016;8(4):418-22.

21 Baş, OT, C; Dede, O; Sarı, S; Çakıcı, MÇ; Öztürk, U; Göktuğ, G; İmamoğlu, A. Factors affecting complication rates of retrograde flexible ureterorenoscopy: analysis of 1571 procedures-a single-center experience. World J Urol. 2016;[Epub ahead of print].

22 Wendt-Nordahl, G, Mut, T., Krombach, P., Michel, M. S. \& Knoll, T. . Do new generation flexible ureterorenoscopes offer a higher treatment success than their predecessors? . Urol Res. 2011(39):185-8.

23 Perlmutter, AEet a. Impact of stone location on success rates of endoscopic lithotripsy for nephrolithiasis. Urology. 2008;71:214-7.

24 Miernik, Aet a. Standardized flexible ureteroscopic technique to improve stone-free rates. . Urology. 2012;80(1198-1202).

25 Rippel, CAet a. Residual fragments following ureteroscopic lithotripsy: incidence and predictors on postoperative computerized tomography. J Urol. 2012;188:2246-51.

26 Portis, AJ, Rygwall, R., Holtz, C., Pshon, N. \& Laliberte, M. Ureteroscopic laser lithotripsy for upper urinary tract calculi with active fragment extraction and computerized tomography followup. J Urol. 2006;175:2129-33.

27 Rebuck, DA, Macejko, A., Bhalani, V., Ramos, P., \& Nadler, RB. The natural history of renal stone fragments following ureteroscopy. Urology. 2011(77):564-8. 
Table 1. Stone locations and treatment

\begin{tabular}{|l|l|}
\hline Patients & $\mathrm{n}=117$ \\
\hline Stone location & \\
\hline Bilateral renal or ureteropelvic junction stones without ureteral stones & $71(60.7 \%)$ \\
\hline Bilateral renal stones with unilateral ureteral stones & $25(21.4 \%)$ \\
\hline Bilateral renal stones with bilateral ureteral stones & $2(1.7 \%)$ \\
\hline Unilateral renal stones and contralateral ureteral stones & $10(8.5 \%)$ \\
\hline Unilateral renal stones, ipsi- and contralateral ureteral stones & $7(6.0 \%)$ \\
\hline Bilateral ureteral stones only & $2(1.7 \%)$ \\
\hline
\end{tabular}

Table 2. Short term patient complications.

\begin{tabular}{|l|l|l|}
\hline & Bilateral & Unilateral \\
\hline Clavien-Dindo I & $\mathbf{n}=\mathbf{1 1}$ & $\mathbf{n}=\mathbf{1 3}$ \\
\hline Stent pain and discomfort & 6 & 8 \\
\hline $\begin{array}{l}\text { Ureteral mucosal erosion without smooth muscle injury } \\
\text { (grade I) }\end{array}$ & 3 & \\
\hline Urge incontinence & 1 & \\
\hline Urinary retention & 1 & 1 \\
\hline Bleeding/ clot retention & & 1 \\
\hline Fevers & & 1 \\
\hline Infection & $\mathbf{n}=\mathbf{4}$ & 2 \\
\hline Clavien-Dindo II & 3 & $\mathbf{n}=\mathbf{5}$ \\
\hline Febrile urinary tract infections & 1 & 5 \\
\hline Acute renal injury & $\mathbf{n}=4$ & $\mathrm{n}=3$ \\
\hline Clavien Dindo III (n=4) & 2 & 1 \\
\hline $\begin{array}{l}\text { Hydronephrosis and intractable flank pain after stent } \\
\text { removal }\end{array}$ & 1 & 1 \\
\hline Laser injury of urothelium (grade II) & 1 & \\
\hline $\begin{array}{l}\text { Ureteral dilation with contrast extravasation in distal ureter } \\
\text { (grade III) }\end{array}$ & 1 & \\
\hline Clavien IV and V (n=0) & & \\
\hline
\end{tabular}

